\title{
Influence of the ultrasonic vibration amplitude on the melt pool dynamics and the weld shape of laser beam welded EN AW-6082 utilizing a new excitation system for laser beam welding
}

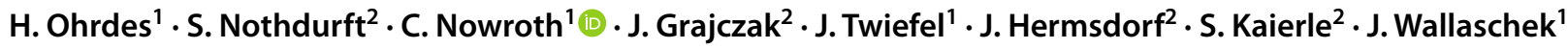

Received: 31 July 2020 / Accepted: 3 December 2020 / Published online: 6 January 2021

(c) The Author(s) 2021

\begin{abstract}
Laser beam welding is a commonly used technology for joining similar and dissimilar materials. In order to improve the mechanical properties of the weld, the introduction of ultrasonic vibration into the weld zone has been proposed [5]. The ultrasonic system consists of an electronic control, a power supply, a piezoelectric converter and a sonotrode, which introduces the vibration into the weld zone. Its proper design is of great importance for the process performance. Furthermore, the effects of ultrasound in a melt pool need to be understood to evaluate and optimize the process parameters. In addition, it is important to find out the limits of ultrasonic excitation with respect to a maximum vibration amplitude. Therefore, firstly different methods of ultrasonic excitation are investigated and compared with respect to their performance. A system which is based on using longitudinal vibrations turns out to be the best alternative. Secondly, the system design is described in detail to understand the boundary conditions of the excitation and finally, simulations about the influence of ultrasonic vibrations are done by using a simplified model. The system is used to perform experiments, which aim at detecting the maximum vibration amplitude doing bead on plate welds of EN AW-6082 aluminum alloy. The experiments reveal a significant change of the weld shape with increasing ultrasonic amplitude, which matches the simulative findings. If the amplitudes are small, there is a marginal effect on the weld shape. If the amplitudes are high, melt is ejected and the weld shape is disturbed. In the present case, amplitudes over $4 \mu \mathrm{m}$ were found to disturb the weld shape.
\end{abstract}

Keywords Ultrasound $\cdot$ Weld shape $\cdot$ Laser beam welding $\cdot$ Excitation methods $\cdot$ Aluminum alloy $\cdot$ Melt pool dynamics

\section{Introduction}

Laser beam welding is a joining technology that uses energy emitted by a laser to weld materials. It has several advantages compared to other fusion welding methods. Due to the localized heat input, narrow welds with welding depths up to $20 \mathrm{~mm}$ [1] and small heat affected zones can be achieved. The process can be automated and it enables joining a variety of different materials.

C. Nowroth

nowroth@ids.uni-hannover.de

$1 \quad$ Institute of Dynamics and Vibration Research, Leibniz University Hannover, An der Universität 1, 30823 Garbsen, Germany

2 Laser Zentrum Hannover E.V., Hollerithallee 8C, 30419 Hannover, Germany
It has been shown that the mechanical properties of the welds can be effected by superimposed vibrations. Campbell [2] and Jose et al. [3] have listed proven effects of vibrations on solidification processes. These include an improvement of the breaking strength, the ultimate tensile strength, the yield strength, and the hardness. All these improvements are caused by finer grains and a more uniform grain distribution. For laser beam welding, a grain refinement can be achieved possibly by acoustic cavitation inside the melt pool, which is stimulated by the ultrasonic vibration, if its amplitude is strong enough. Cavitation goes hand in hand with strong local pressure fluctuations in the melt. The dynamic pressure change effects small, hollow, high-energy bubbles. At sufficiently high pressure amplitudes, the bubbles implode and release their energy, which effects very high local temperatures and strong local streams as a result of shock waves. Jiang et al. [4] investigated the effects of the excitation frequency on the microstructure, the mechanical properties, and the fracture behavior of A356 aluminum alloy obtained 
by expendable pattern shell casting. They found that the grain size decreased with increasing frequency. Additionally, the tensile strength, yield strength, elongation, and hardness increased due to the vibration. Tewari [5] investigated the influence of transverse vibrations at frequencies between 0 and $400 \mathrm{~Hz}$ and vibration amplitudes between 0 and $40 \mu \mathrm{m}$ on an arc welding process. The study concluded that grain refinement is caused by dendrite fragmentation. The influence of the ultrasonic amplitude on the weld seam properties has been investigated in [6]. The extent to which local excitation influences the weld seam was also investigated. The local excitation depends on the excited vibration shape of the system and, in particular, on the relative position of the weld with respect to the vibration nodes and antinodes of the system. In [6], the situation where the melt bath is in a vibration anti-node (which means that the entire melt bath is shaken) was compared with the situation where the melt bath is in a vibration node (which means that the borders of the melt bath are expanded and compressed). The ultrasonic frequency used was $20 \mathrm{kHz}$. It was found that higher vibration amplitudes and an excitation near the vibration anti-node leads to the best grain refinement. Since high frequencies and high amplitudes lead to high pressures, it seems reasonable to excite the melt with higher frequencies, e.g. ultrasonic vibrations [2]. Zhoua et al. [7] excited nickel-based alloy and austenite stainless steel ultrasonically during a laser beam welding process. The excitation direction was parallel to the laser. They concluded that the excitation leads to better melt mixing in the melt pool. Krajewski et al. [8] investigated the influence of ultrasonic vibrations on an arc-welding process of aluminum alloys. The arc-welding process was used to create a weld seam on ultrasonically excited workpieces that were attached directly to the transducer of the ultrasonic system. Grain refinement and a reduction of porosity were achieved. Furthermore, no mechanical decohesion of the weld-face occurred when welding in a vibration node. The vibration had a negative influence on the quality, when the weld was at a vibration anti-node. A limitation, however, existed in the experimental setups: the samples were usually screwed to the transducer and the position in the vibration distribution was changing during the processes [10]. To overcome this problem, the developed system is capable of butt welding round samples at approximately $20 \mathrm{kHz}$ with an adaptable welding position in the vibration distribution at different ultrasonic vibration amplitudes. Different excitation methods are discussed in this paper. In a previous work [9], it was shown that a similar setup, a longitudinal ultrasonic vibration system with a small water filled fluid container in a vibration anti-node, leads to high fluid dynamics. Another limitation in the previous studies concerns the restrictions of the excitation level. The amplitudes used in the experiments were rather small. However, since higher amplitudes have a higher influence on the microstructure, a high excitation amplitude is of major interest. This, however, leads to the question if there is a critical excitation amplitude leading to lower qualities. Additionally, the excitation can lead to a change in the weld seam shape. In [10], the change of the bead geometry in laser beam welds was investigated. A change was observed; but the experiments had been limited to low power ultrasound and a small welding depth.

The present contribution focuses on both finding the limits of the excitation amplitude and understanding the dynamic processes in the molten pool while doing bead on plate welds of an aluminum alloy. The resulting seam geometry for vibration amplitudes of up to $8 \mu \mathrm{m}$ is investigated experimentally and the observed effects are discussed with the support of a simplified fluid dynamics model of the melt pool.

The system design and a discussion on the excitation methods are provided in Sect. 2. Subsequently the experimental setup and the simulation model are described in Sect. 3 and Sect. 4, respectively. In Sect. 5, the results of the experiments and simulations are presented and combined.

\section{System design}

The system design is presented to discuss the introduction of ultrasonic energy into the melt pool regarding following boundary conditions. The welding process is an ultrasonic assisted laser beam welding process and intended to join two round metal bars with diameters of $30 \mathrm{~mm}$. The laser beam is positioned at the interface of the two work pieces and melts both materials. The energy input leads to a dynamic melt behavior, whereby the melt pool is mixed. In order to improve the mixing and refine the resulting microstructure, the melt pool should be excited ultrasonically. To design a system for this purpose, several requirements have to be taken into consideration. First, the ultrasonic vibration needs to be introduced properly into the work pieces so that the melt pool can be excited. To prevent the system from clattering at the force fitted connections of the work pieces, those must be clamped with sufficiently high forces. In addition, the work piece holder must be able to rotate the work pieces, as the laser beam cannot be rotated for safety reasons. Finally, all the subsystems (rotation system, ultrasound generation, laser system, work piece holder) need to be controlled simultaneously. All subsystems and their integration are described in the following subsections. The subsequent welding tests to prove the system are bead on plate welds of the aluminum alloy EN AW-6082, because it is widely used in industry and it is sensitive to ultrasound due to its low melt viscosity. 


\subsection{Ultrasonic system}

\subsubsection{Excitation methods}

There are different possible methods to assist the laser beam welding process by ultrasonic vibrations. All these methods have advantages and disadvantages. In this section, the different methods are discussed and appraised. Based on the rating, the most appropriate method is selected.

2.1.1.1 Fluid coupling One possible method to excite the melt pool is excitation through a fluid as coupling medium. An ultrasonic transducer sends high energy ultrasonic waves through a coupling medium to the melt pool, as seen in Fig. 1. An advantage of this method is its easy adaptability to different specimen geometries with frequency change by changing the ultrasonic transducer only. Disadvantages are the need for a coupling medium and the high loss of ultrasonic intensity. The coupling medium should be a fluid that is capable of transmitting the ultrasound from the transducer to the melt pool. The low efficiency is caused by the impedance differences between transducer, coupling medium and work piece. The ultrasonic wave generated in the transducer must first be transferred into the coupling medium and then transferred from the coupling medium to the specimen and the melt pool. At each surface the waves are reflected due to the impedance steps, which causes intensity loss of the transmitted wave. Further, the system needs to be capsulated due to the fluid, which increases the designing effort for the integral system. For optimal placement, the laser beam and the ultrasonic transducer should be collocated. (For example, a hollow transducer allowing the passing of the laser beam.)

2.1.1.2 Structure coupling Another concept is the structure coupling where the welded specimens are integral parts of the vibration system. To drive such a setup most efficiently at frequencies in the ultrasonic range, it is controlled to perform vibrations in one of its fundamental resonance fre-

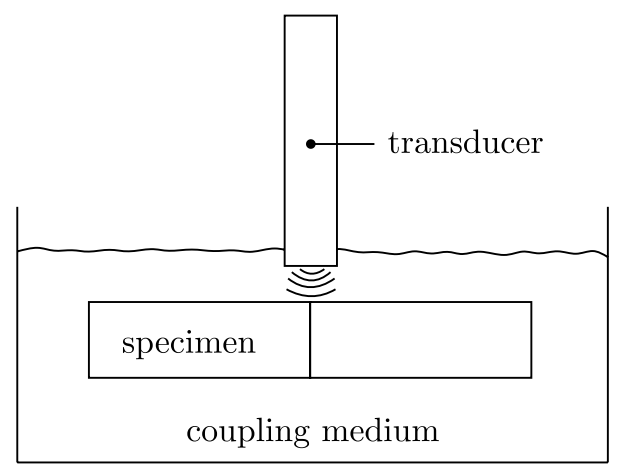

Fig. 1 Fluid coupling concept quencies. In this configuration, different vibration modes can be considered. The vibration system can be excited in a bending, torsional, or longitudinal mode. For all modes, it is possible to weld in the vibration maximum, the minimum, or in-between. As a result, these concepts offer manifold possibilities for various excitations.

The common advantages of the three depicted modes (Fig. 2a-c) are good impedance matching due to the direct coupling as well as the possibility to locate the melt pool in different positions of the mode shape.

The bending mode (Fig. 2a) has some major disadvantages, which exclude this concept from further consideration. One disadvantage is the mounting concept of the vibration system. Since the work pieces need to be force fitted in the beginning of the joining process, very high axial preload is needed. Therefore, the system needs to be axially clamped, causing high damping at the bearing since there is no plane without vibration existing in the bending mode. Another disadvantage is the non-uniform excitation around the specimens that is critical in order to rotate the work piece. The excitation direction would change from perpendicular vibration to parallel vibration to the laser radiation with all combinations in-between. Hence, this setup directly leads to a non-uniform influence on the melt pool.

The torsional mode (Fig. 2b) does not possess the aforementioned disadvantages. The bearing of the system is an achievable design task and the excitation is constant along the circumference. The disadvantage of this concept, however, is that the vibration amplitude is not constant over the welding depth. The maximal amplitudes are located at the specimen surface and no vibration exists in the specimen center.

A third excitation method is the longitudinal mode, Fig. 2c. Similar to the torsional concept, the bearing is an achievable task. Additionally, the vibration is constant in amplitude and direction along the circumference and along the welding depth. Furthermore, the welding process can be

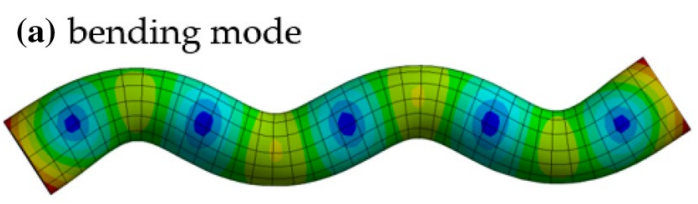

(b) torsional mode

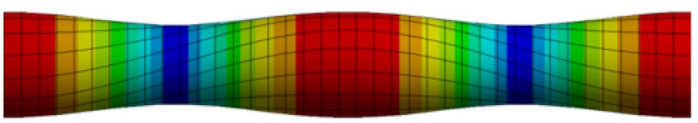

(c) longitudinal mode

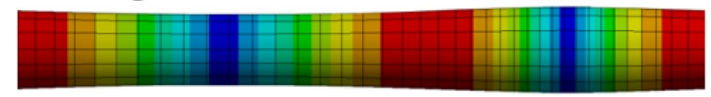

Fig. 2 Structure coupling concepts 
performed in a vibration node, an anti-node, or anywhere inbetween, which makes this system very versatile for different excitation methods.

All advantages and disadvantages of the different excitation methods are summarized in Table 1. With these characteristics, the longitudinal mode appears to be the most suitable concept and will be utilized in the next steps of this study.

\subsubsection{Ultrasonic system design}

The main purpose of the ultrasonic system is to introduce ultrasonic energy into the weld pool. A longitudinal vibration mode is selected to excite the weld pool at different positions in the vibration distribution. The selected resonance frequency is $20 \mathrm{kHz}$. It is approximately the lowest frequency in an inaudible range. Much higher frequencies than $20 \mathrm{kHz}$ lead to the problem that more power has to be applied for the same vibration amplitude. Further, a lower frequency is tied along with a longer wavelength, and therefore there are less wavelengths in a system with constant length. This again is important since the damping effect increases with the number of wavelengths. In order to reach a reasonable vibration amplitude, boosters are designed and applied to amplify the vibration amplitude. The system consists of several parts that all are designed individually to vibrate at a half of the driving wavelength $(\lambda / 2)$, including the work pieces, as shown in Fig. 3. The converter (boltedclamped-transducer) is used to transform electrical energy into mechanical vibration energy. The generated vibration amplitude is then amplified by the two subsequent boosters. The first booster has an additional bearing plate that is used to introduce the clamping force into the vibration system. Subsequent to the boosters, an adapter specifically designed for the following work piece is connected, so that the two parts combined are a $\lambda / 2$ part. The following $\lambda / 2$ parts are identical parts in a mirrored assembly. In this configuration, the second set of boosters decreases the vibration amplitude to have a minimum vibration amplitude at the second bearing plate.

Table 1 Advantages and disadvantages of the different excitation methods (A: indirect coupling by a fluid, B: direct coupling - bending mode, C: direct coupling - torsional mode, D: direct coupling - longitudinal mode)

\begin{tabular}{lcccc}
\hline & $\mathrm{A}$ & $\mathrm{B}$ & $\mathrm{C}$ & $\mathrm{D}$ \\
\hline Impedance matching & - & + & + & + \\
Frequency flexibility & + & - & - & - \\
Excitation position flexibility & - & + & + & + \\
Bearing & + & - & + & + \\
Uniform excitation & $\mathrm{o}$ & - & - & + \\
\hline
\end{tabular}

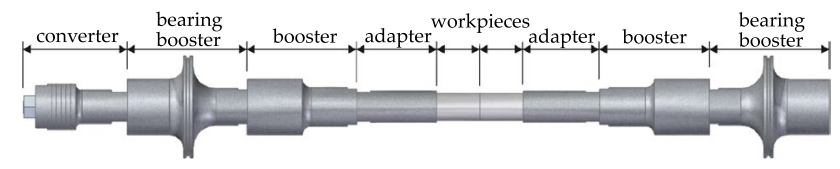

Fig. 3 Designed ultrasonic vibration system

The design of all individual parts and the integral system is defined using FEM-simulation in Ansys Workbench. The result of the design process is shown in Fig. 4. It depicts the deflection of the operational longitudinal vibration mode and illustrates that the design has the highest vibration amplitude at the interface between the work pieces and the lowest vibration amplitude at the bearing plates, which is the desired vibration state.

\subsection{Clamping system}

The clamping system is essential because the work pieces have neither a fixed connection to each other nor to the vibration system. The second reason for clamping is to prevent the system from detaching during the vibration. To calculate the required clamping force that prevents the system from detaching, a calculation for a simplified system is used [11]. Assuming a uniform rod type system operated in a longitudinal resonance, the maximum occurring stress, which is at the vibration node, can be calculated according to (1)

$\hat{T}_{\text {max }}=|\rho c \hat{v}|=\left|2 \pi \rho c f_{r} \hat{x}\right|$

where $\hat{T}_{\max }$ is the maximum stress amplitude, $\rho$ is the material's density, $\hat{v}$ is the vibration velocity amplitude at antinodal position, $\hat{x}$ is the corresponding vibration amplitude and $f_{r} \approx 20 \mathrm{kHz}$ is the resonance frequency. The longitudinal wave propagation velocity $c$ is calculated by (2) [12]

$c=\sqrt{\frac{E}{\rho} \frac{(1-v)}{\left(1-v-2 v^{2}\right)}}$

where $v$ is Poisson's ratio and $E$ is Young's modulus. All parts of the vibration system are made of titanium except the work pieces to be welded. These are made of steel $(20 \mathrm{MnCr} 5)$ or aluminum (EN AW-6082). Assuming a

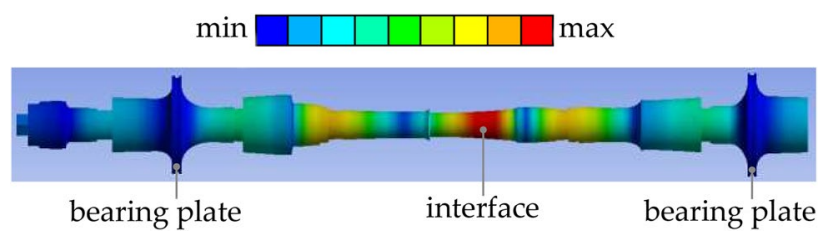

Fig. 4 Vibration distribution of the ultrasonic system in the modal analysis 
Table 2 Significant values for the dimensioning of the vibration system

\begin{tabular}{llll}
\hline Material & $\mathrm{C}[\mathrm{m} / \mathrm{s}]$ & $\hat{T}_{\text {max }}[\mathrm{MPa}]$ & $F_{\text {max }}[\mathrm{kN}]$ \\
\hline Ti6Al-4 V (3.7165) & 6100 & 100 & 72 \\
EN AW-6082 (3.2315) & 6250 & 64 & 45 \\
20MnCr5 (1.7147) & 5850 & 170 & 120 \\
\hline
\end{tabular}

constant density and a constant wave propagation velocity and using the material parameters and a vibration amplitude of $\hat{x}=30 \mu \mathrm{m}$, the values for the maximum stress amplitude are calculated. Compared with the other two materials, steel leads to the highest maximum stress amplitude shown in Table 2.

Thus the resulting force that is needed to prevent the system from detaching using work pieces made of steel is calculated by

$F_{\text {max }}=\hat{T}_{\text {max }} A \approx 120 \mathrm{kN}$

where $A$ is the cross-section area of the clamped work pieces with a diameter of $30 \mathrm{~mm}$.

To provide this force, a hydraulic system with three parallel hydraulic cylinders is used. Springs with different degrees of stiffness are prestressed by the cylinders to guarantee the needed clamping force in the system in the case of work piece shrinkage during the process. Different springs are mounted on each side of four shafts that are used to guide the vibration system. Since the spring stiffness at each side is different, a nonlinear spring characteristic is achieved. First, softer springs are prestressed to provide a sufficient spring deflection. The softer springs are mounted in caps, which act as stops after $8.3 \mathrm{~mm}$ displacement, where they provide a force of $25 \mathrm{kN}$. Then, the stiffer springs provide a force of up to $390 \mathrm{kN}$ before they reach their end stop at a displacement of additional $1.7 \mathrm{~mm}$. Since the load to the system is rather high, it has to be ensured that no buckling can occur. Therefore, a buckling load calculation in Ansys Workbench is performed. For the calculation, one bearing plate of the vibration system is fixed and the other one is loaded axially.

The simulation estimates a critical clamping force of

$F_{\text {crit. }} \approx 650 \mathrm{kN}$

Since $F_{\text {crit. }}$ is much higher than $F_{\max }$, buckling is not expected.

\subsection{Rotary system}

The rotary system consists of two rotary bearings for the rotary degree of freedom and a motor to rotate the system automatically. Since the system prestress is high, the axial load capacity of the bearings must likewise be high enough to withstand the force. Therefore, two angular contact ball bearings of type 7226 are used in o-assembly. Due to the high normal force, the bearing torque reaches a value of up to $16 \mathrm{Nm}$. To provide this torque, a gear motor with a nominal torque of $1 \mathrm{Nm}$ and a gear stage with a ratio of 1:25 are used. Subsequently, a gear stage with two gears and a ratio of 13:21 amplifies the torque to the needed value and transmits the torque to the ultrasonic system. This setup is able to provide up to $40 \mathrm{Nm}$ of torque to the vibration system, which is sufficient to rotate the system even at high clamping loads.

\subsection{System for ultrasonic assited laser beam welding}

The integral system consists of three plates. Two plates are clamping the ultrasonic system and the third one is needed to apply the force to it. All plates are mounted on four shafts that serve as linear guidance and transmission of the clamping force. Since the clamping force is quite high, a stress analysis for all parts is done as a basis for the needed dimensions. The constructed setup is depicted in Fig. 5.

\subsection{Laser beam welding system}

The laser beam source used is a diode-pumped, solid-state disk laser with a maximum output power of $16 \mathrm{~kW}$. The laser power is fed into the welding head by an optical fiber with a $200 \mu \mathrm{m}$ diameter. With the welding head optics the resulting focal diameter is $400 \mu \mathrm{m}$. For the welding process, a laser power $P_{L}$ of $4.5 \mathrm{~kW}$ and a welding speed $v_{F}$ of $1.85 \mathrm{~m} / \mathrm{min}$ result in an energy per unit length of $259.5 \mathrm{~J} / \mathrm{cm}$. The incidence angle of the beam is set as $10^{\circ}$ to avoid damage to the optical system by back reflection.

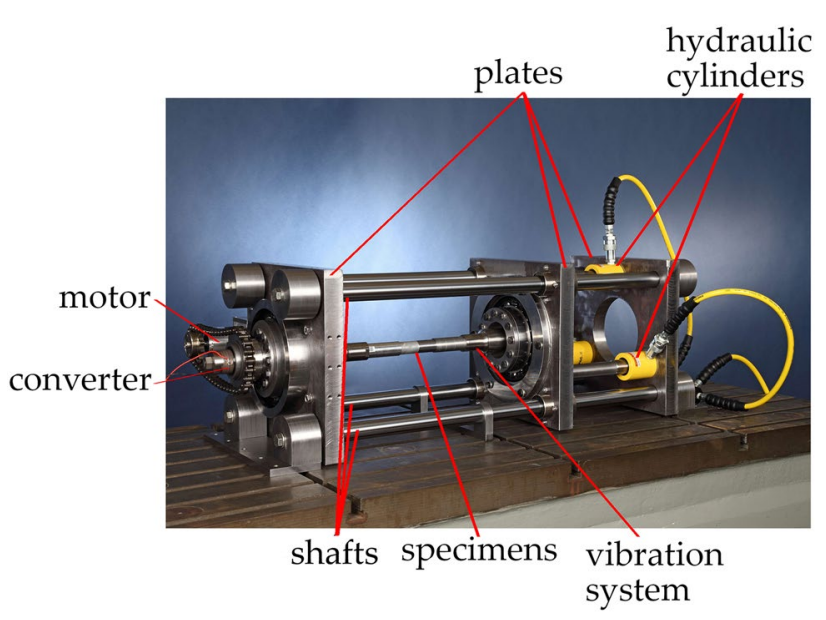

Fig. 5 System for ultrasonic assisted laser beam welding 


\subsection{Control of the ultrasonic vibrations and data acquisition}

Multiple control units are applied to control the corresponding parts of the system. The ultrasonic system is closed-loop controlled by an in house developed digital phase controller (DPC500/100 k) [13, 14]. This system uses the voltage and current that are measured at the transducer with a voltage probe (Testec TT-SI 9010) and a current probe (Tektronix P6021A) as inputs. From these, the current amplitude and the phase between current and voltage are detected. The DPC500/100 k also allows to compensate the parallel capacitance of the piezo elements [13]. By using this compensation, the system characteristic of the mechanical system can be detected directly. This also allows the operation of medium damped ultrasonic systems. Furthermore, the compensated current is directly proportional to the vibration amplitude. The system then controls the phase and compensated current amplitude to a set value. The output is amplified by an amplifier (QSC RMX 5050a) and a subsequent transformer (Self-made 1:6) to match the impedance and provide the power needed for the process. The motor (XBP086409-02) is driven by an AMI1023-01 controller both from the company Ott $\mathrm{GmbH} \& \mathrm{Co}$. KG. Both the position and the rotation speed can be controlled. Doing this simultaneously offers various advantages for the welding process, e. g. a constant laser energy per unit length due to constant rotation speed and defined start/stop positions. Both ultrasound and rotation systems are externally operated by a main software running on a PC. In the software, a program sequence is set so that all components can be driven in a desired order and for a desired duration. For safety reasons, the laser components are running using its independent system. Therefore, a KUKA-system is used that controls the inert gas and the laser beam. This system is triggered by a signal initiated by the main software utilizing the motor control unit. The laser is switched off after a previously calculated duration corresponding to one rotation cycle of the samples. All data needs to be stored for further evaluation. Therefore, the main software in combination with a USB-oscilloscope (PicoScope 3406D) is used to log the high frequency (sample rate $1 \mathrm{MS} / \mathrm{s}$ ) data of the current, voltage and the laser trigger. The recording is again triggered by a signal generated by the laser system before the start and after the end of the laser process. Further, the motor controller is used to log the rotation angle and speed. After acquisition, the data from the motor unit and the USB-oscilloscope are synchronized using the trigger signal that activates the laser system, which is sent from the motor unit and is logged by the PicoScope.

\section{Experimental setup}

\subsection{Evaluation of the vibration shape}

To prove the functionality of the system, the ultrasonic system is first analyzed. The longitudinal vibration distribution between the boosters, especially near the intended position of the melt pool, is measured. For this test, the system is driven at resonance with a low vibration amplitude (low driving current) by the DPC500/100 k. The vibration velocity is measured by a differential fiberoptic laser vibrometer in an in-plane setup that makes it possible to measure inplane velocities. Additionally, the vibration amplitude at the vibration maximum is measured at different driving currents to evaluate the dependency between vibration amplitude and current.

\subsection{Weld seam shape experiments}

To evaluate the influence of the ultrasonic excitation on the weld seam shape, bead on plate welds (welding in monomaterial) in aluminum alloy EN AW-6082 are performed. For this, an aluminum alloy shaft is clamped into the system and the focal point of the laser is set to a vibration maximum. At this position, multiple welds at different ultrasonic vibration amplitudes, $0 \mu \mathrm{m}$ (without ultrasound) as a reference, 2, 4, 6 and $8 \mu \mathrm{m}$ are performed. Subsequently, the samples are prepared for metallographic cross sections to evaluate the resulting weld seam shapes.

\section{Simulation of the vibration influence}

To gain understanding of the fluid dynamics in the melt pool, a simplified model of this pool is proposed. The main target is to evaluate the influence of the ultrasonic vibration on the melt pool. For this, a 2D model is used (Fig. 6) that consists of a rectangular container filled with fluid. The chosen fluid has the properties of molten aluminum that are similar to the properties of water with a density of $998 \mathrm{~kg} / \mathrm{m}^{3}$ and a viscosity of $0,001 \mathrm{~kg} / \mathrm{ms}$. The upper area is filled with air with its properties at room temperature. All parameters represent the properties of the materials during the welding process and were assumed to be constant for the simulation. The upper area enables the melt to move outside the molten pool. The model is solved using the volume of fluid method included in Ansys Fluent. The excitation is realized by moving both sidewalls of the fluid container with a certain amplitude at a frequency of $20 \mathrm{kHz}$. Both walls vibrate in phase, which simulates an excitation in a vibration maximum. All effects induced directly by the laser beam energy, namely the 


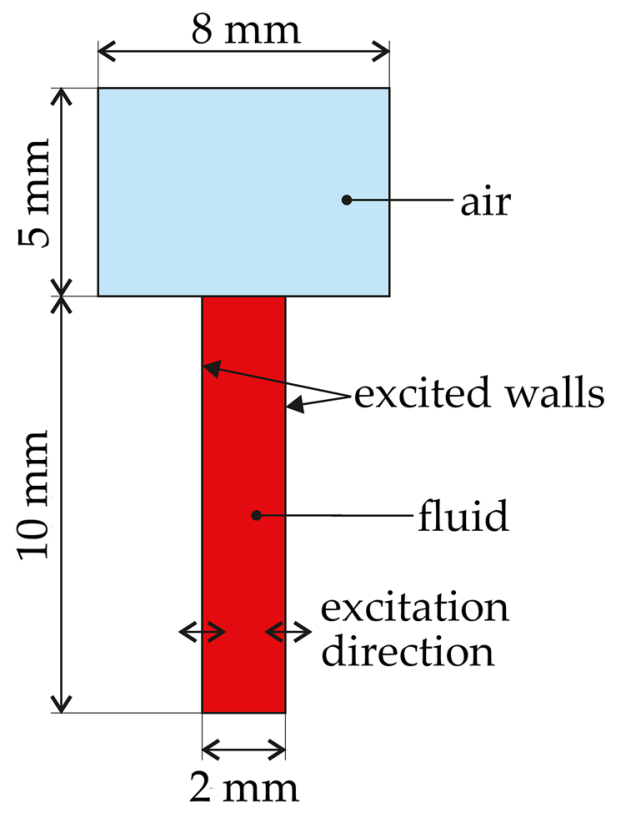

Fig. 6 Scheme of the simplified melt pool model

keyhole generation, the vapor pressure, or the fluid dynamics caused by melting, are neglected. With this simplified model, the influence of ultrasound on the fluid is separated, which will provide a first insight on the ultrasound induced fluid dynamics in the melt pool.

\section{Results and discussion}

\subsection{System evaluation}

The vibration distribution measurement closely resembles the simulated distribution. As expected, the vibration maximum is positioned between the work pieces, see Fig. 7. The dependency between current and vibration amplitude is measured and found to be slightly nonlinear. The higher the driving current, the lower the vibration amplitude per current is.

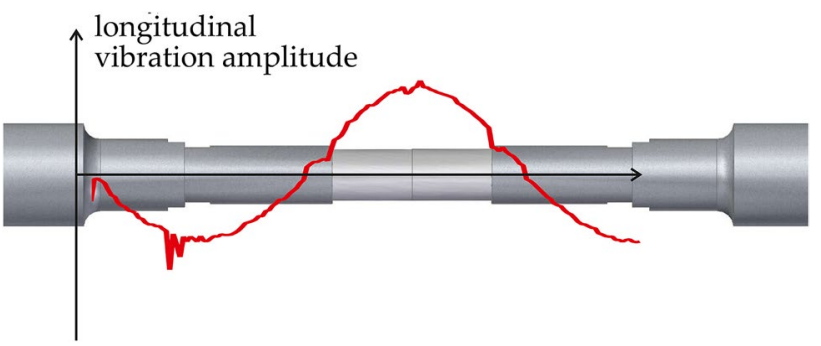

Fig. 7 Measured vibration distribution of the ultrasonic system in the area of interest
Without considering the slight nonlinearity, the dependency can be approximated as

$\hat{x} \approx 10 \frac{\mu \mathrm{m}}{\mathrm{A}} \hat{i}$

where $\hat{x}$ is the vibration amplitude and $\hat{i}$ is the current amplitude. The vibration amplitudes for the following discussions are calculated using this approximation.

\subsection{Weld seam shape evaluation}

The experiments aim to evaluate the influence of the ultrasonic vibration amplitude on the resulting weld seam shape, the limits of the excitation amplitude, and the underlying mechanisms. Fig. 8 depicts the change of the weld seam shape for different ultrasonic vibration amplitudes by metallographic cross sections. The shape without ultrasonic excitation is the typical shape for a laser deep penetration welding process. With increasing ultrasonic vibration amplitude, there is sagging in the middle and reinforcement at the edges. At higher vibration amplitudes starting at $6 \mu \mathrm{m}$, the sagging in the middle becomes clearly V-shaped. Additionally, the seam root changes to a round shape and the overall shape differs from the typical shape. Furthermore, the weld depth decreases for the investigated amplitudes higher than $4 \mu \mathrm{m}$.

In Fig. 9, the shapes are sketched to distinguish between the different phenomena. For the rising edges, sagging of the middle and V-shape collapse phenomena can be explained by the hypothesis described by the ultrasonically induced
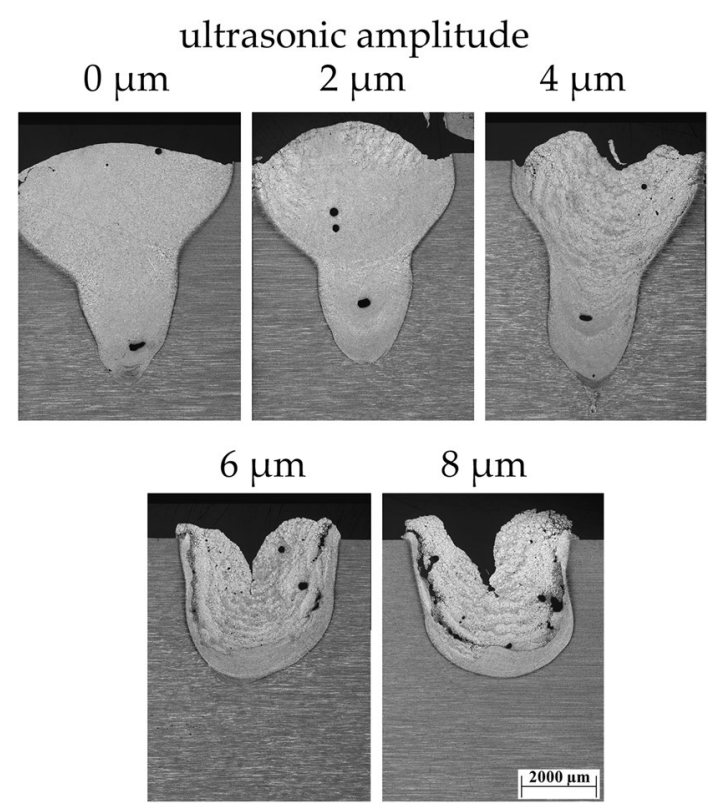

Fig. 8 Metallographic cross sections of the weld seams for different ultrasonic amplitudes 


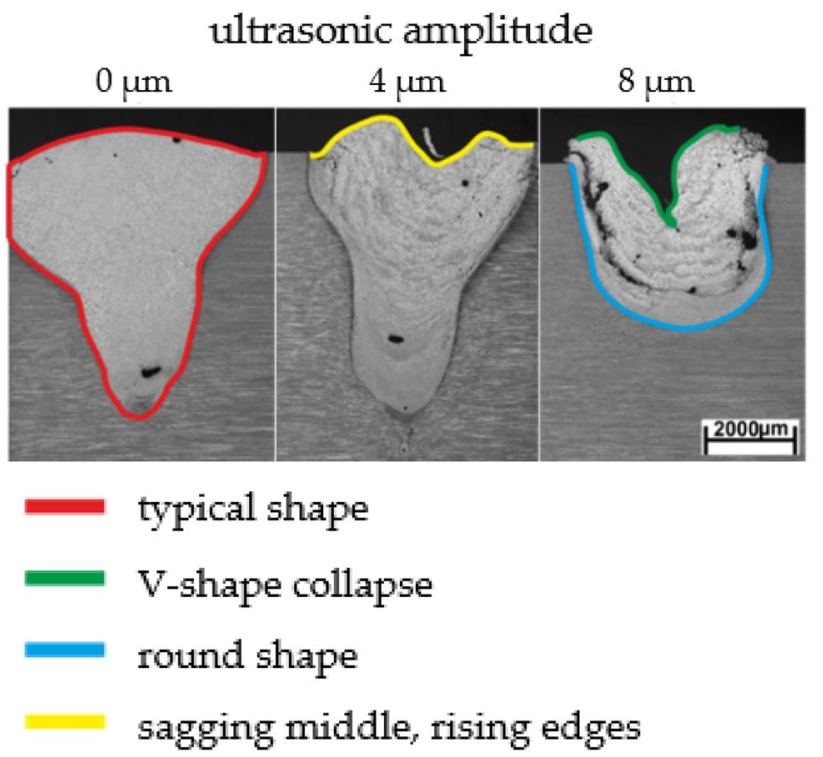

Fig. 9 Metallographic cross sections of the weld seams for different ultrasonic amplitudes with emphasized attributes

melt pool dynamics. This will be investigated by the simulative analysis. The round shape and decrease of the weld depth results from an interaction between the ultrasonically induced fluid dynamics and the laser induced melt pool dynamics. Possible reasons for the round shape and the decreasing depth are either the collapse of the keyhole or an incompletely generated keyhole. Both scenarios could have been caused by an extremely low local laser energy level, which could be caused by ejected melt drops or a disruption of the arising keyhole by the ultrasound induced fluid dynamics.

\subsection{Simulative findings}

The model described in Sect. 4 is used to simulate the influence of ultrasonic excitation on the simplified melt pool at different vibration amplitudes. Fig. 10 depicts the effects of different excitation levels by showing the resulting steady

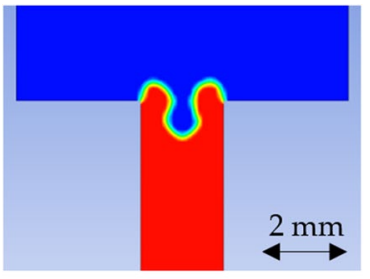

(a) $2 \mu \mathrm{m}$

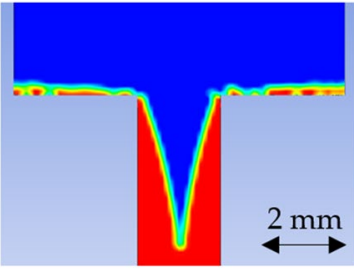

(b) $10 \mu \mathrm{m}$
Fig. 10 Simulation results for different excitation levels at steady state after $300 \mathrm{~ms}$

state of the fluid in the melt pool. At low excitation levels, the fluid rises up at the top edges and sinks in the middle (Fig. 10a). This behavior is similar to the excitation level of $4 \mu \mathrm{m}$ amplitude in the experiment. At higher excitation levels (Fig. 10b), the fluid shows a clear V-shaped collapse in the middle, which is also similar to the experimental results at an excitation level of 6 and $8 \mu \mathrm{m}$. This demonstrates that the model provides matching qualitative results to the experiments and is an indicator that the ultrasonic induced fluid dynamics is sufficient to describe the effects on the weld shape.

The reason for rising edges and sinking middle is the fluid dynamics induced by the ultrasound. When a wall moves to the inside, the inert melt will not move immediately in the same direction, but is instead accelerated upwards. This can be observed at the left pool boundary in Fig. 11a, where this pool boundary moves to the inside and displaces the melt to the top. The fluid on the right pool boundary that moves to

moving right:

(a)

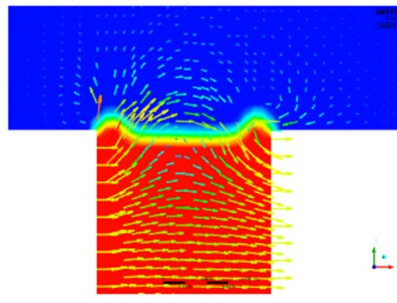

(d)

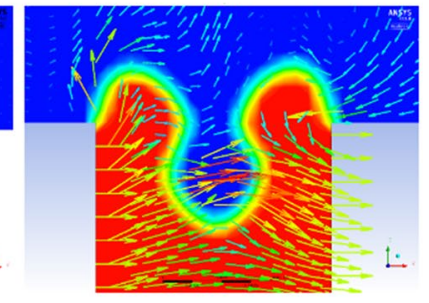

turning point: (b)

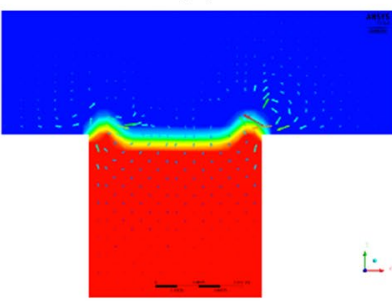

moving left:

(c)

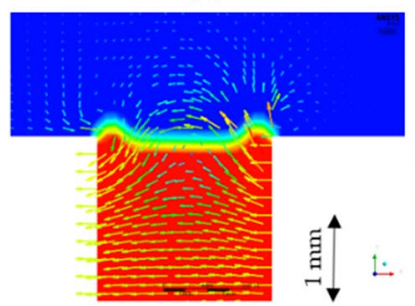

(e)

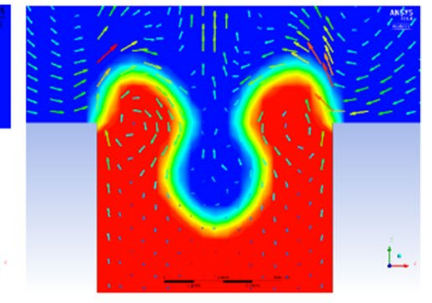

(f)

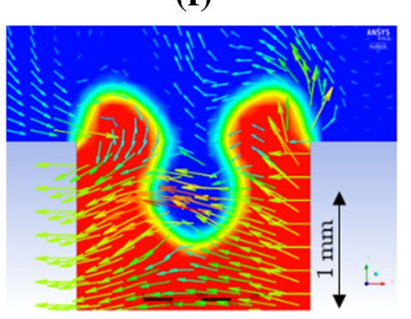

Fig. 11 Velocity vectors for starting transient a-c and steady state d-f conditions, $2 \mu \mathrm{m}$ amplitude 


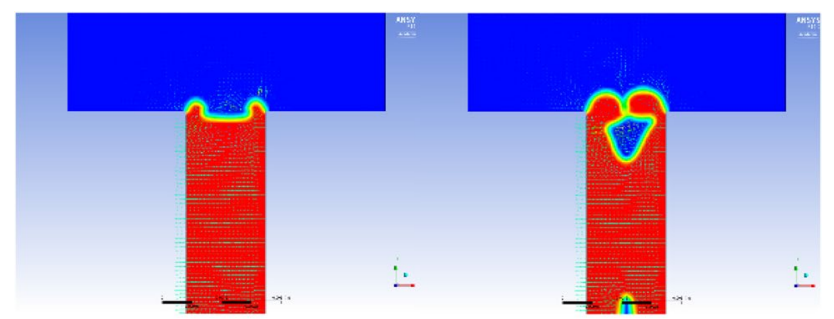

(a) $1 \mathrm{~ms}$

(b) $5 \mathrm{~ms}$

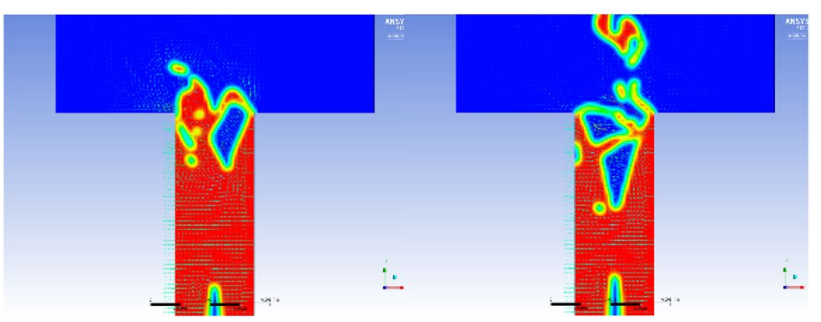

(c) $10 \mathrm{~ms}$

(d) $20 \mathrm{~ms}$

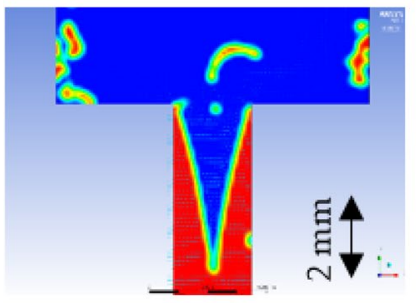

(e) $70 \mathrm{~ms}$

Fig. 12 Simulation results for $10 \mu \mathrm{m}$ excitation at different time steps

the outside is accelerated to the bottom as depicted by the velocity vectors in Fig. 11. The resistance at the bottom is remarkably higher than that at the top. This is due to the melt and the pool boundary itself providing a higher resistance against the movement of the melt than the air over the melt pool. Thus, a mean movement of the melt in the upper direction results from the alternatingly moving pool boundaries. For small excitation amplitudes, the acceleration at the top equals the gravity before the fluid gets ejected or touches itself in the middle, reaching a steady state (Fig. 11d-f). Due to mass conservation, the melt in the pool middle sinks down, represented by the velocity vectors in Fig. 11a-c.

If a certain excitation level is exceeded, the bulges at each corner touch each other in the middle, as in Fig. 12b. At this point, the steady eddy in these bulges is disrupted and the melt starts to eject due to the induced turbulence (Fig. 12cd). After the chaotic phase, the amount of melt at the top corners is small enough to be ejected rather fast due to the moving pool boundaries. This finally leads to a steady state (Fig. 12e). At this point, the V-shape that is similar to the experiment has been formed.

With this simple model, two of the occurring phenomena in the experiment can be described. Furthermore, a possible reason for the round shape at the melt pool bottom can be derived. As observed in Fig. 12b, the fluid closes the existing hole, which could also lead to a closing keyhole in the experiment. The keyhole can also be disrupted by the vibrating melt surrounding the keyhole or by spattering melt as observed in Fig. 12c, d. Another influence on the keyhole stability can be an ultrasonically altered melt flow. Such a disrupted or even closed keyhole needs to be reopened by the laser beam, which takes time and energy that is not available for deeper penetration.

\section{Conclusions}

A new system for ultrasonically assisted laser beam deep penetration welding with high ultrasonic amplitudes up to $8 \mu \mathrm{m}$ was designed, built and its functionality was proved. The system was used to investigate the influence of strong ultrasonic excitation on the resulting weld seam shape of aluminum alloy EN AW-6082. For this, the maximum possible vibration amplitude without negatively influencing the weld seam shape was detected by simulations and experiments. Vibration amplitudes of up to $4 \mu \mathrm{m}$ did not change the typical weld shape. At higher excitation amplitudes $(>6 \mu \mathrm{m})$, the melt was ejected from the molten bath, which leads to a loss of material and therefore to a reduced connection area and thus, to a lower joint strength. The proposed melt pool model helps to understand that at lower vibration amplitudes, the melt is balanced by the equilibrium between gravity and ultrasonically induced forces. At this excitation level, the melt is mixed well by the ultrasound, but still stays in the molten bath. At higher amplitudes, the rising corners touch each other, closing the hole and ejecting melt chaotically. The investigation identifies a critical ultrasonic excitation amplitude for the tested aluminum alloy. Exceeding that amplitude causes a major negative influence on the weld seam shape. For investigations on the influence on the microstructure of ultrasonically assisted laser beam welded aluminum alloy, please see [6]. There, ultrasonic excitation causes a decreasing and more homogenous grain size, decreasing weld depth and width, but increasing width/depth ratio, as well as a different porosity distribution.

In future investigations, other materials and material combinations consisting of aluminum, different steel grades and nickel-base alloys as well as other welding positions in the vibration distribution will be tested with this new setup. In result, more information about the exact interaction mechanisms between ultrasound and welding area including keyhole should be achieved.

Acknowledgements The authors would like to thank the German Research Foundation (DFG) for the financial and organisational support of this project. 
Author contributions Conceptualization: H. Ohrdes, S. Nothdurft, J. Twiefel, J. Hermsdorf, S. Kaierle, J. Wallaschek; Methodology: H. Ohrdes, S. Nothdurft, C. Nowroth Formal analysis and investigation: H. Ohrdes, S. Nothdurft, C. Nowroth; Writing - original draft preparation: H. Ohrdes, S. Nothdurft; Writing-review and editing: C. Nowroth, J. Grajczak, J. Twiefel, J. Hermsdorf, S. Kaierle, J. Wallaschek; Funding acquisition: S. Kaierle, J. Wallaschek; Resources: H. Ohrdes, S. Nothdurft, C. Nowroth; Supervision: S. Kaierle, J. Wallaschek; Data curation: H. Ohrdes, S. Nothdurft, C. Nowroth; Visualization: H. Ohrdes, C. Nowroth, S. Nothdurft; Project administration: S. Nothdurft, J. Twiefel, J. Hermsdorf.

Funding Open Access funding enabled and organized by Projekt DEAL. Funded by the Deutsche Forschungsgemeinschaft (DFG, German Research Foundation) - CRC 1153, subproject A3-252662854.

\section{Compliance with ethical standards}

Conflict of interest The authors declare that they have no conflict of interest.

Open Access This article is licensed under a Creative Commons Attribution 4.0 International License, which permits use, sharing, adaptation, distribution and reproduction in any medium or format, as long as you give appropriate credit to the original author(s) and the source, provide a link to the Creative Commons licence, and indicate if changes were made. The images or other third party material in this article are included in the article's Creative Commons licence, unless indicated otherwise in a credit line to the material. If material is not included in the article's Creative Commons licence and your intended use is not permitted by statutory regulation or exceeds the permitted use, you will need to obtain permission directly from the copyright holder. To view a copy of this licence, visit http://creativecommons.org/licenses/by/4.0/.

\section{References}

1. Rominger V (2011) High-performance laser welding a comparison of $\mathrm{CO} 2$ lasers and high-brilliance solid-state lasers. Laser Tech J $8(3): 32-35$

2. Campbell J (1981) Effects of vibration during solidification. Int Mater Rev 2:71-108
3. Jose MJ, Kumar SS, Sharmai A (2019) Vibration assisted welding processes and their influence on quality of welds. Sci Technol Weld Joining 21:243-258

4. Jiang W, Chen X, Wang B, Fan Z, Wu H (2016) Effects of vibration frequency on microstructure, mechanical properties, and fracture behavior of A356 aluminum alloy obtained by expendable pattern shell casting. Int J Adv Manuf Technol 83:167-175

5. Tewari SP (1999) Effects of Transverse Oscillation on Tensile Properties of Mild Steel Weldments. ISIJ Int 39(6):570-574

6. Nothdurft S, Ohrdes H, Twiefel J, Wallaschek J, Mildebrath M, Maier HJ, Hassel T, Overmeyer L, Kaierle S (2019) Influence of ultrasonic amplitude and position in the vibration distribution on the microstructure of a laser beam welded aluminum alloy. J Laser Appl 31(2):022402

7. Zhoua S, Maa G, Wua D, Chaia D, Lei M (2018) Ultrasonic vibration assisted laser welding of nickel-based alloy and Austenite stainless steel. J Manuf Processes 31:759-767

8. Krajewski A, Wlosinski W, Chmielewski T, Kolodziejczak P (2012) Ultrasonic-vibration assisted arc-welding of aluminum alloys. Bull Pol Acad Sci 60(4):841-852

9. Ohrdes H, Fischer H, Twiefel J, Wallaschek J (2016) Experimental investigation of ultrasonic fluid mixing as assistance for a laser welding process. Proceedings of International Conference on Noise and Vibration Engineering, Leuven

10. Venkannah S, Mazumder J (2009) Changes in Laser Weld Bead Geometry with the Application of Ultrasonic Vibrations. Proceedings of World Congress on Engineering, London

11. Neppiras EA (1960) Very high energy ultrasonics. Br J Appl Phys $11: 143-150$

12. Kuttruff H (1988) Physik und Technik des Ultraschalls. S. Hirzel, Stuttgart

13. Twiefel J, Klubal M, Paiz C, Mojrzisch S, Krüger H (2008) Digital signal processing for an adaptive phase-locked loop controller. Proc SPIE Int Soc Opt Eng 6929:69260A-A69261

14. Ille I, Twiefel J (2015) Model-based feedback control of an ultrasonic transducer for ultrasonic assisted turning using a novel digital controller. Physics Procedia 70:63-67

Publisher's Note Springer Nature remains neutral with regard to jurisdictional claims in published maps and institutional affiliations. 